\title{
Diabetes mellitus e sua relação com a COVID-19: um panorama atual proveniente de
}

\section{uma revisão sistemática}

\author{
Diabetes mellitus and its relationship with COVID-19: A current overview from a systematic review \\ Diabetes mellitus y su relación con COVID-19: resumen actual de una revisión sistemática
}

Recebido: 31/10/2021 | Revisado: 10/11/2021 | Aceito: 17/11/2021 | Publicado: 28/11/2021

Bianca Sampaio Lima

ORCID: https://orcid.org/0000-0002-0574-4936 Instituto de Educação Superior do Vale do Parnaíba, Brasil E-mail: biancasp1234@gmail.com

Maiza Carneiro Machado Frota

ORCID: https://orcid.org/0000-0002-3666-2749 Instituto de Educação Superior do Vale do Parnaíba, Brasil E-mail: maiza.carneiro@ hotmail.com

Sara Prado Ramos

ORCID: https://orcid.org/0000-0001-8736-4621 Instituto de Educação Superior do Vale do Parnaíba, Brasil E mail:saraprado124@gmail.com

José Lopes Pereira Júnior

ORCID: https://orcid.org/0000-0001-5621-7469 Instituto de Educação Superior do Vale do Parnaíba, Brasil E-mail: josejrfarmaceutico@gmail.com

Antonio de Pádua Rocha Nóbrega Neto

ORCID: https://orcid.org/0000-0003-4829-0062 Instituto de Educação Superior do Vale do Parnaíba, Brasil E-mail: antonio.neto@iesvap.edu.br

\begin{abstract}
Resumo
Introdução: A ocorrência de Diabetes Mellitus (DM), vêm se elevando gradativamente, o que se torna uma preocupação global. Atualmente, esta preocupação se deve ao fato de alguns estudos sugerirem que o diabetes está associado a um maior risco de desenvolver formas graves de COVID-19. Objetivo: Realizar uma revisão sistemática acerca da DM e sua relação com COVID-19. Metodologia: Trata-se de um estudo de revisão sistemática da literatura utilizando as bases de dados PubMed (Biblioteca Nacional de Medicina dos Estados Unidos) e Science Direct com os descritores "Diabetes mellitus", "covid-19", "fatores de risco/Diabetes mellitus", "covid-19", "comorbidade". A pesquisa foi limitada com base na data de publicação (no tempo de 2 anos: 2019-2021), espécies (humanos), idiomas disponíveis em (inglês, espanhol e português). Foram excluídos das pesquisas os artigos que não faziam parte da temática "Diabetes Mellitus e sua relação com a COVID-19. Resultados e Discussão: Durante o estudo foi possível observar a forte correlação entre o mal prognóstico de COVID-19 e DM tipo 1 ou 2. Visto que o diabetes imunocompromete o corpo humano, entendese que uma infecção secundária a COVID-19 seja mais persistente e letal. Conclusão: Conclui-se que pacientes portadores de DM que contraem COVID-19 apresentam um pior prognóstico quando comparados a pacientes não diabéticos. Esses pacientes devem receber uma maior atenção do sistema de saúde, recebendo medidas preventivas como um rigoroso controle glicêmico. Urge também mostrar a essa população a importância da vacinação.
\end{abstract}

Palavras-chave: Diabetes mellitus; COVID-19; Comorbidade.

\begin{abstract}
Introduction: Currently, the occurrence of type 2 Diabetes Mellitus (DM2) is increasing gradually, which becomes a global concern. This concern is due to the fact that some studies suggest that diabetes is associated with an increased risk of developing severe forms of COVID-19. Objective: To conduct a systematic review of DM in relation to COVID19. Methodology: This is a systematic literature review study using the PubMed (United States National Library of Medicine) and Science Direct databases with the descriptors "Diabetes mellitus", "covid-19", "risk factors / Diabetes mellitus", " covid-19", “c comorbidity". The search was limited based on publication date (5 years' time: 2016-2021), species (humans), available languages (English, Spanish and Portuguese). Articles that were not part of the COVID-19 $x$ Diabetes Mellitus theme were excluded from the research. Results and Discussion: During the study, it was possible to observe the strong correlation between the poor prognosis of COVID-19 and Diabetes type 1 and 2. Since diabetes immunocompromises the human body, it is understood that an infection secondary to COVID - 19 is more persistent and lethal. Conclusion: It is concluded that patients with diabetes who contract have a worse prognosis when compared to non-diabetic patients. These patients should receive greater attention from the health system, receiving preventive measures such as strict glycemic control. It is also urgent to show this population the importance of vaccination.
\end{abstract}


Keywords: Diabetes mellitus; COVID-19; Comorbidity.

\begin{abstract}
Resumen
Introducción: En la actualidad, la ocurrencia de Diabetes Mellitus tipo 2 (DM2) ha ido aumentando paulatinamente, lo que se convierte en una preocupación mundial. Esta preocupación se debe al hecho de que algunos estudios sugieren que la diabetes está asociada con un mayor riesgo de desarrollar formas graves de COVID-19. Objetivo: Realizar una revisión sistemática de la DM y su relación con COVID-19. Metodología: Se trata de un estudio de revisión sistemática de la literatura utilizando las bases de datos PubMed (Biblioteca Nacional de Medicina de los Estados Unidos) y Science Direct con los descriptores "Diabetes mellitus", "covid-19", "factores de riesgo / Diabetes mellitus", "covid-19", "comorbilidad". La búsqueda fue limitada según la fecha de publicación (tiempo de 5 años: 2016-2021), especies (humanos), idiomas disponibles (inglés, español y portugués). Los artículos que no formaban parte del tema COVID19 x Diabetes Mellitus fueron excluidos de la investigación. Resultados y Discusión: Durante el estudio se pudo observar la fuerte correlación entre el mal pronóstico de COVID-19 y Diabetes tipo 1 y 2 dado que la diabetes inmunodeprime el cuerpo humano, se entiende que una infección secundaria a COVID-19 es más persistente y letal. Conclusión: Se concluye que los pacientes con diabetes que contraen covid tienen peor pronóstico en comparación con los pacientes no diabéticos. Estos pacientes deben recibir una mayor atención por parte del sistema de salud, recibiendo medidas preventivas como un estricto control glucémico. También es urgente mostrar a esta población la importancia de la vacunación.
\end{abstract}

Palabras clave: Diabetes mellitus; COVID-19; Comorbilidad.

\title{
1. Introdução
}

No final do ano de 2019 ocorreu um surto de doença coronavírus 2019 (COVID-19) em Wuhan, na China (Cheng et al., 2021). Dentre as principais características do coronavírus, encontra-se seu RNA envelopado (Chan et al., 2020) e um dos maiores genomas virais (Cheng et al., 2021). O mesmo é pertencente ao subtipo do gênero beta e está relacionado à família do coronavírus com síndrome respiratória aguda grave (SARS-CoV), desse modo, passou a ser chamado também de SARS-CoV2. A atual pandemia global está relacionada a este vírus, o que resultou no aumento de casos e fatalidades em todo o mundo, com mais de três milhões de casos relatados em 5 de maio de 2020 (Chan et al., 2020). Ao se pensar na infecção do COVID19, o período médio de incubação é de aproximadamente 2 a 5 dias, e quanto às manifestações clínicas as mais comuns são: febre $(89,6 \%)$, tosse $(69,9 \%)$, dispneia $(59,6 \%)$ e fadiga $(52,3 \%)$ no início da doença e alguns pacientes também apresentam anorexia $(35,2 \%)$ e diarreia $(26,4 \%)$ (Cheng et al., 2021), (Yan et al., 2020).

Já a diabetes, é uma doença crônica que acomete grande parte da população mundial. Dessa forma, com o advento da pandemia do Sars-CoV-2, muito se especulou sobre o impacto do vírus na população diabética.

Desse modo, desde o início da pandemia em 2020 foram realizados vários estudos enfatizando a relação dos casos de internação por COVID-19 e sua relação com outras comorbidades, sendo a hiperglicemia crônica uma das principais pelo seu comprometimento à resposta imune, resultando em várias discussões que revelam os riscos de internação a partir de casos de pacientes com comorbidades pregressas.

Assim, quando se contextualiza a incidência da diabetes, em especial a DM Tipo 2 (DM2), é de rápido crescimento, especialmente em pessoas que estão em fase de envelhecimento (Cheng et al., 2021). Pacientes com DM de longa data, constantemente apresentam outras comorbidades, como hipertensão, obesidade, doenças cardiovasculares e doença renal crônica. (Chan et al., 2020). O DM2 pode ser identificado com base nos códigos do Sistema de Classificação Internacional de Doenças (CID-10) ou com qualquer resultado de $\mathrm{HbA1c} \geq 6,5 \%$ ou prescrição de anti-hiperglicêmicos nos últimos doze meses. (Satman et al., 2021). Visto que um paciente com DM apresenta hiperglicemia, o sistema inato e imunológico encontra-se enfraquecido e desregulado, limitando a capacidade do corpo humano de resistir a qualquer infecção. (Cheng et al., 2021).

Visto isso, em meio às internações por COVID-19, em vários países evidencia-se a grande quantidade de pessoas com diabetes em qualquer idade e sexo, sendo este considerado um fator predisponente para o aumento de mortes intra-hospitalares e em sua totalidade. (Yan et al., 2020). 
A grande preocupação da maior ocorrência de DM na população, se deve ao fato de alguns estudos sugerirem uma associação a um maior risco de desenvolver formas graves de COVID-19 (Al-Salameh et al., 2021). Um estudo nacional do Reino Unido evidenciou que o DM tipo 1 e o tipo 2 foram associados ao aumento significativo na taxa de mortalidade relacionada ao COVID-19. Além disso, este aumento da mortalidade, foi associado às complicações de ambos os tipos de diabetes (Cheng et al., 2021).

A explicação desse agravamento de pacientes com DM e COVID-19, se baseia em estudos que evidenciam que estes pacientes sofrem de respostas inflamatórias mais graves e apresentam menor quantidade de linfócitos do que os pacientes não diabéticos. Os pacientes acometidos pelo COVID-19 e com DM2 por exemplo, a inflamação e os linfócitos se resolvem mais lentamente e a hiperglicemia, a linfopenia e a inflamação desempenham papéis importantes na gravidade de COVID-19 com diabetes. De acordo com estudos realizados, em comparação com o grupo não diabético, os pacientes com COVID-19 com DM2 que possuem a idade mais avançada possuem maior risco de casos graves, além dos diabéticos terem a exacerbação de resposta inflamatória com aumento de níveis de leucócitos, de contagem de neutrófilos, proteína de reação C de alta sensibilidade, procalcitonina, ferritina, receptor de interleucina (IL) 2, IL-6, IL-8, fator de necrose tumoral $\alpha$, D-dímero, fibrinogênio, desidrogenase láctica e peptídeo natriurético $\mathrm{N}$-terminal pró-cérebro e baixos linfócitos. Com isso, os pacientes com maior risco de morte são homens, os quais apresentam resposta inflamatória grave, disfunção pulmonar com a redução do volume expiratório forçado, capacidade vital forçada, associado cardiopatia, doença hepática, renal e de coagulação. Desse modo, o período de sobrevida de pacientes com e sem DM foi de 10 e 18 dias. (Yan et al., 2020).

Sabe- se, que com o panorama da pandemia, é necessário um cuidado mais abrangente em virtude das variantes de risco para COVID-19, principalmente nos cuidados com diabéticos adaptando as medidas de prevenção necessárias e informando as estratégias de vacinação em massa (McGurnaghan et al., 2021). Diante disso, o objetivo desse estudo foi apresentar um panorama atual acerca da DM e sua relação com a COVID-19 através de uma revisão sistemática.

\section{Metodologia}

Trata-se de um estudo de revisão sistemática da literatura, realizada em cinco (05) etapas, tais como: (1) a definição da questão de pesquisa e identificação das bases de dados para consulta, (2) estabelecimento dos critérios de elegibilidade e busca na literatura de potenciais estudos primários elegíveis, (3) análise e avaliação da elegibilidade dos estudos, (4) extração dos dados relevantes, (5) discussão da síntese dos resultados e apresentação do estudo.

Com base na temática abordada e no objetivo do estudo a questão norteadora da pesquisa, foi elaborada utilizando a estratégia do acrônimo PICO (População = Seres Humanos, intervenção/exposição = Infectados por coronavírus/ diabetes mellitus; comparação = Infectados por coronavírus e desfecho = Fator de risco ), e a partir dos elementos do acrônimo, elencamos os descritores utilizados na busca de literatura e definimos a pergunta norteadora: "Como a diabetes mellitus pode ser um fator de risco para pacientes infectados pelo Sars-CoV-2?"

As buscas de estudos elegíveis foram realizadas no período de 5 de abril de 2021 a 12 de abril de 2021, nas bases de dados: Biblioteca Nacional de Medicina dos Estados Unidos (PubMed) e Science Direct, por três pesquisadores, de maneira concomitante e independente, utilizando os elementos oriundos do acrônimo PICO para obtenção de descritores específicos e controlados, disponíveis no Descritores em Ciências da Saúde/Medical Subject Headings (DeCS/MeSH): Diabetes mellitus, covid 19, fatores de risco/Diabetes mellitus, comorbidade os quais foram combinados durante a busca pelos operadores booleanos AND e OR.

Estabeleceram-se como critérios de inclusão: Relatos de Casos, Estudo Clínico, Ensaio Clínico, Estudo Observacional, Ensaio Randomizado Controlado, disponíveis em inglês, espanhol e português, atribuindo-se o recorte de tempo de 2 anos (2019-2021). Em seguida, os artigos selecionados foram submetidos a uma segunda análise, onde foi realizada 
a leitura do texto completo, contemplando, assim, os artigos que realmente se adequaram aos critérios de inclusão, à metodologia e à temática, excluindo-se os fora de contexto e os duplicados.

\section{Resultados e Discussão}

De acordo com a Figura 1, foram eleitos 264 artigos para a leitura do resumo, 89 artigos que abordaram a temática foram selecionados e 175 artigos que não condiziam com o propósito deste estudo foram excluídos. Obteve-se uma segunda análise resultando em 36 artigos que preenchiam os critérios propostos e foram seguidos, nessa ordem, os seguintes passos: leitura do texto completo e análise dos textos aos que se adequavam ao tema proposto.

A partir da análise dos textos, foi elaborado o Quadro 1, que expõe os autores, nacionalidades, população, objetivo, tipo de estudo/método e resultados nos artigos selecionados com o objetivo de sistematizar e facilitar a comparação entre as abordagens das diversas fontes.

Figura 1. Análise dos artigos selecionados.

Fonte: Autores.

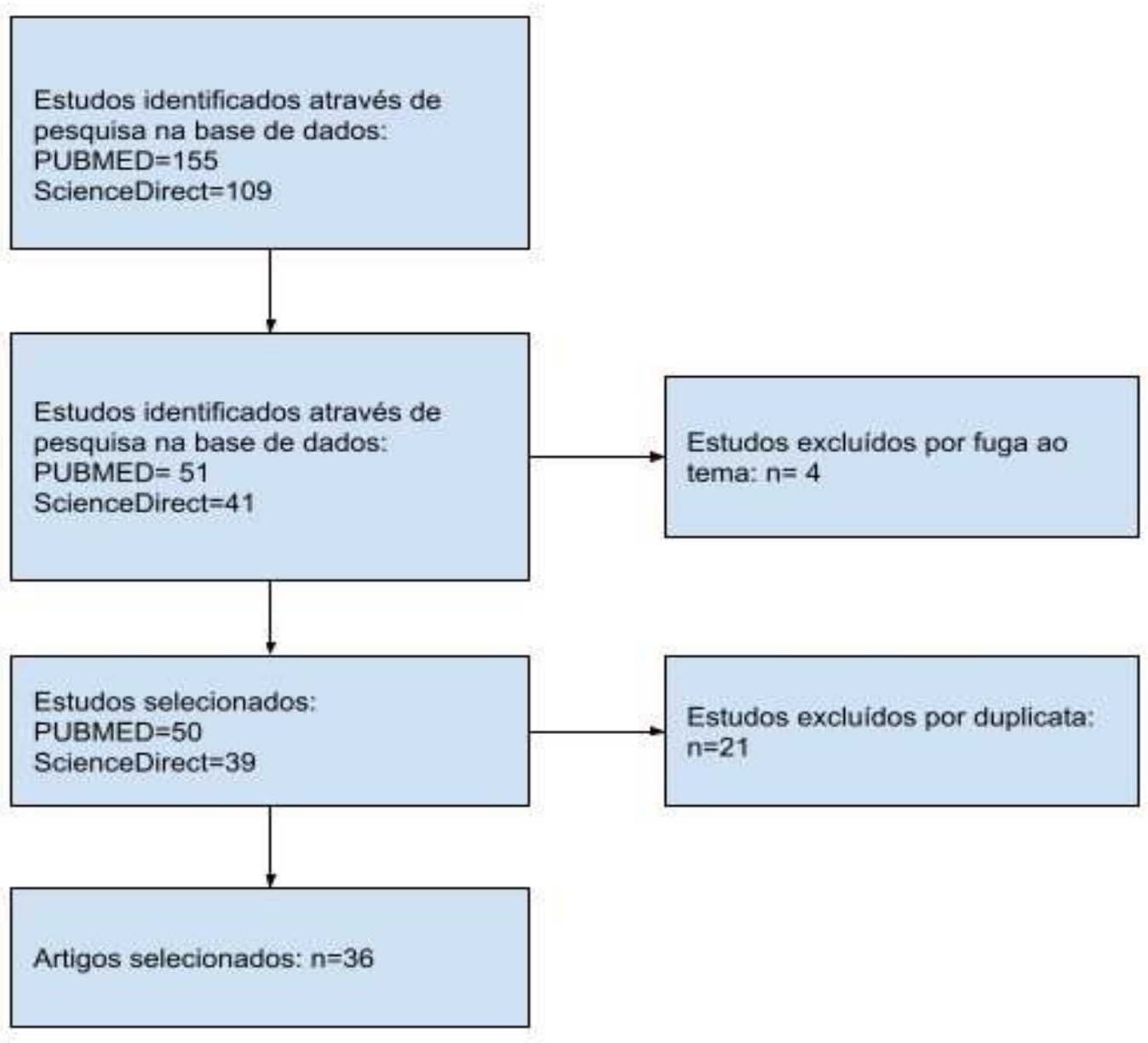


Research, Society and Development, v. 10, n. 15, e384101522598, 2021

(CC BY 4.0) | ISSN 2525-3409 | DOI: http://dx.doi.org/10.33448/rsd-v10i15.22598

Quadro 1. Análise descritiva dos estudos incluídos.

\begin{tabular}{|c|c|c|c|c|c|}
\hline Autor & Nacionalidade & População & Objetivo & $\begin{array}{c}\text { Tipo de } \\
\text { estudo/método }\end{array}$ & Resultados \\
\hline $\begin{array}{l}\text { Abbas et } \\
\text { al., } 2021\end{array}$ & Bagdá, Iraque & $\begin{array}{l}284 \text { pacientes COVID-19 } \\
\text { envolvendo pacientes saudáveis, } \\
\text { fumantes e diabéticos }\end{array}$ & $\begin{array}{l}\text { Comparar o risco adverso grave do tabagismo } \\
\text { com as comorbidades e resultados do diabetes } \\
\text { em pacientes com COVID- } 19 \text {. }\end{array}$ & $\begin{array}{c}\text { Estudo } \\
\text { descritivo }\end{array}$ & $\begin{array}{l}\text { Os sinais mais comuns da doença foram febre e tosse. Além disso, os pacientes } \\
\text { do sexo masculino eram mais vulneráveis à infecção do que as do sexo feminino. } \\
\text { Múltiplas comorbidades estão diretamente associadas à gravidade da progressão } \\
\text { da doença por COVID-19 e a uma maior taxa de mortalidade. Fumantes se } \\
\text { apresentaram como casos leves em comparação com diabéticos e fumantes mais } \\
\text { diabéticos, que se apresentaram como casos graves a críticos. }\end{array}$ \\
\hline $\begin{array}{l}\text { Akter et } \\
\text { al., } 2020\end{array}$ & Bangladesh & $\begin{array}{l}\text { Total de } 734 \text { pacientes, das quais } \\
19,8 \% \text { dos pacientes tinham } \\
\text { diabetes e } 76 \% \text { dos pacientes } \\
\text { COVID-19 eram do sexo } \\
\text { masculino. }\end{array}$ & $\begin{array}{l}\text { Investigar as manifestações clínicas, resultados } \\
\text { e complicações de longo prazo de pacientes } \\
\text { internados com COVID-19 na parte sul de } \\
\text { Bangladesh, enfatizando os indivíduos com } \\
\text { diabetes. }\end{array}$ & $\begin{array}{l}\text { Estudo } \\
\text { transversal }\end{array}$ & $\begin{array}{l}\text { Indivíduos com diabetes experimentaram manifestações mais grave de COVID- } \\
19 \text { e complicações pós- doença. }\end{array}$ \\
\hline $\begin{array}{l}\text { Akbariqom } \\
\text { i et al., } \\
2020\end{array}$ & Irã, Teerã & $\begin{array}{l}595 \text { pacientes internados por covid } \\
19 \text { com e sem diabetes entre } 55 \\
\text { anos e homens. }\end{array}$ & $\begin{array}{l}\text { Descrever as características epidemiológicas e } \\
\text { clínicas juntamente com os desfechos de } \\
\text { pacientes hospitalizados com Doença do } \\
\text { Coronavírus } 2019 \text { (COVID-19) com e sem } \\
\text { diabetes. }\end{array}$ & $\begin{array}{l}\text { Estudo } \\
\text { retrospectivo }\end{array}$ & $\begin{array}{l}\text { Acometidos de diabetes apresentaram maiores chances de complicações e } \\
\text { mortalidade em comparação ao grupo sem diabetes. }\end{array}$ \\
\hline $\begin{array}{l}\text { Alam et } \\
\text { al., } 2021\end{array}$ & Bangladesh & $\begin{array}{l}\text { Um total de } 780.961 \text { pessoas } \\
\text { preencheram a pesquisa online e, } \\
\text { entre elas, } 1.516 \text { pessoas foram } \\
\text { diagnosticadas para COVID- } 19 \text {. }\end{array}$ & $\begin{array}{l}\text { Investigar principalmente informações } \\
\text { demográficas, de morbidade, clínicas e } \\
\text { diagnósticas relacionadas ao COVID-19 de um } \\
\text { punhado de participantes e, entre eles, } 1.516 \\
\text { pessoas foram diagnosticadas como COVID } \\
\text { positivo (+) e comparar pacientes (casos) } \\
\text { positivos para COVID com idade, sexo e com } \\
\text { situações de comorbidade crítica. }\end{array}$ & $\begin{array}{l}\text { Pesquisa de } \\
\text { autorrelato }\end{array}$ & $\begin{array}{l}\text { Dois terços dos participantes do estudo são do sexo masculino e, além da } \\
\text { obesidade, o número de outras comorbidades é maior nos casos de COVID- } 19 \text {, } \\
\text { a partir da regressão logística uni variada, participantes do sexo feminino e } \\
\text { pessoas com } 60 \text { anos ou mais apresentaram maior risco de COVID-19 e } \\
\text { associações de risco significativamente mais elevadas foram observadas para a } \\
\text { idade, sexo feminino, diabetes, doença renal. }\end{array}$ \\
\hline $\begin{array}{l}\text { Al- } \\
\text { Salameh } \\
\text { et al., } \\
2021\end{array}$ & Amiens, França & $\begin{array}{l}\text { Quatrocentos e trinta e seis } \\
\text { pacientes, incluindo homens, de } \\
\text { idade média de } 72 \text { anos. }\end{array}$ & $\begin{array}{l}\text { Comparar as características clínicas e os } \\
\text { resultados em pacientes com COVID-19 } \\
\text { hospitalizados com e sem diabetes }\end{array}$ & $\begin{array}{l}\text { Estudo de caso } \\
\text { controle, } \\
\text { observacional }\end{array}$ & $\begin{array}{l}\text { Diabetes foi associado a um maior risco de admissão na UTI e um tempo de } \\
\text { internação mais longo, mas não com mortalidade (relativo a pacientes não } \\
\text { diabéticos). }\end{array}$ \\
\hline $\begin{array}{l}\text { Alzaid et } \\
\text { al., } 2020\end{array}$ & Paris, França & 45 pacientes com COVID-19 & $\begin{array}{l}\text { Relacionar a gravidade da COVID-19 e a } \\
\text { linfocitopenia } \\
\text { sistêmica. }\end{array}$ & $\begin{array}{l}\text { Estudo } \\
\text { observacional }\end{array}$ & $\begin{array}{l}\text { COVID-19 grave está associado à linfocitopenia de células CD8 na população } \\
\text { em geral, enquanto a monocitopenia de monócitos clássicos é específica para } \\
\text { pacientes com DM2. }\end{array}$ \\
\hline $\begin{array}{l}\text { Arango- } \\
\text { Guerra et } \\
\text { al., } 2021\end{array}$ & $\begin{array}{l}\text { Medellín, } \\
\text { Colombia }\end{array}$ & $\begin{array}{l}15 \text { pacientes que foram internados } \\
\text { para COVID- } 19\end{array}$ & $\begin{array}{l}\text { Identificar se a crise } \quad \text { hiperglicêmica } \\
\text { relacionada à cetoacidose diabética e estado } \\
\text { hiperosmolar não cetótico, pode ser precipitada } \\
\text { por COVID- } 19\end{array}$ & $\begin{array}{c}\text { Estudo } \\
\text { observacional }\end{array}$ & $\begin{array}{l}\text { Observou-se que comorbidades como o diabetes podem estar associadas a formas } \\
\text { mais graves da doença e maiores taxas de mortalidade. }\end{array}$ \\
\hline $\begin{array}{l}\text { Barron et } \\
\text { al., } 2020\end{array}$ & $\begin{array}{l}\text { Londres, Reino } \\
\text { Unido }\end{array}$ & $\begin{array}{l}61414470 \text { indivíduos foram } \\
\text { registrados }\end{array}$ & $\begin{array}{l}\text { Identificar a associação que a insuficiência } \\
\text { cardíaca e a doença cerebrovascular possuem } \\
\text { sobre a desfechos graves relacionados ao } \\
\text { COVID- } 19 \text {. }\end{array}$ & $\begin{array}{c}\text { Estudo } \\
\text { observacional }\end{array}$ & $\begin{array}{l}\text { Resultados mostram um risco aumentado em pessoas com diabetes, com um terço } \\
\text { de todas as mortes hospitalares com COVID-19 ocorrendo em pessoas com } \\
\text { diabetes. }\end{array}$ \\
\hline
\end{tabular}


Research, Society and Development, v. 10, n. 15, e384101522598, 2021

(CC BY 4.0) | ISSN 2525-3409 | DOI: http://dx.doi.org/10.33448/rsd-v10i15.22598

\begin{tabular}{|c|c|c|c|c|c|}
\hline $\begin{array}{l}\text { Bhatti et } \\
\text { al., } 2020\end{array}$ & $\begin{array}{l}\text { Dubai, Emirados } \\
\text { Árabes Unidos }\end{array}$ & 103 pacientes & $\begin{array}{l}\text { Descrever as características clínicas e os } \\
\text { desfechos de pacientes com diabetes internados } \\
\text { em nosso hospital para tratamento com } \\
\text { COVID- } 19 \text {. }\end{array}$ & $\begin{array}{l}\text { Estudo } \\
\text { transversal e } \\
\text { observacional }\end{array}$ & $\begin{array}{l}\text { A maioria dos pacientes com diabetes ou pré-diabetes e COVID- } 19 \text { apresentavam } \\
\text { outras comorbidades notáveis. Nas análises laboratoriais revelaram padróes } \\
\text { anormais distintos de biomarcadores que foram associados a um prognóstico } \\
\text { desfavorável: os níveis de fibrinogênio, dímero D, ferritina e proteína C reativa } \\
\text { foram significativamente mais elevados na admissão em pacientes que } \\
\text { posteriormente necessitaram de cuidados intensivos do que requeles que } \\
\text { necessitaram de enfermaria. }\end{array}$ \\
\hline $\begin{array}{l}\text { Chan et } \\
\text { al., } 2020\end{array}$ & Espanha & Seis pacientes homens & $\begin{array}{l}\text { Explorar o grau de controle do açúcar no } \\
\text { sangue e seu impacto na gravidade da infecção } \\
\text { em pacientes com COVID-19. Necessita-se } \\
\text { dissecar a interação entre o SARS- CoV-2 e o } \\
\text { sistema da angiotensina pancreática, para que } \\
\text { possamos ter um melhor entendimento sobre a } \\
\text { patogênese da hiperglicemia induzida por } \\
\text { SARS-CoV-2 e DKA e HHS combinados. }\end{array}$ & $\begin{array}{l}\text { Estudo } \\
\text { retrospectivo } \\
\text { observacional }\end{array}$ & $\begin{array}{l}\text { É fundamental ter um controle rigoroso da glicose tanto no ambiente hospitalar } \\
\text { quanto no ambulatorial, ao lidar com pacientes diabéticos. Outro achado notável } \\
\text { desta série de casos é que a maioria dos pacientes apresentou sintomas de CAD / } \\
\text { HHS em vez de sintomas respiratórios, e SARS-CoV-2 foi detectado } \\
\text { principalmente porque nossa instituição estava no epicentro da pandemia e todas } \\
\text { as admissões foram rastreadas para COVID-19. }\end{array}$ \\
\hline $\begin{array}{l}\text { Chen et } \\
\text { al., } 2020\end{array}$ & Zhejiang, China & 145 pacientes & $\begin{array}{l}\text { Investigar as características clínicas do } \\
\text { COVID-19 em Zhejiang, China. }\end{array}$ & $\begin{array}{l}\text { Estudo } \\
\text { observacional } \\
\text { retrospectivo de } \\
\text { centro único }\end{array}$ & $\begin{array}{l}\text { Pacientes mais velhos ou pacientes com comorbidades, como obesidade ou } \\
\text { diabetes mellitus, eram mais propensos a ter uma condição grave. Os tratamentos } \\
\text { com COVID-19 ainda são experimentais e mais ensaios clínicos são necessários. }\end{array}$ \\
\hline $\begin{array}{l}\text { Cheng et } \\
\text { al., } 2021\end{array}$ & Wuhan & $\begin{array}{l}\text { Duzentos e trinta e seis pacientes, } \\
\text { incluindo homens e mulheres }\end{array}$ & $\begin{array}{l}\text { O estudo visa a identificação de relação entre a } \\
\text { gravidade de pacientes com COVID-19 e com } \\
\text { diabetes mellitus tipo } 2 \text { (DM2). }\end{array}$ & $\begin{array}{l}\text { Estudo de caso- } \\
\text { controle }\end{array}$ & $\begin{array}{l}\text { Os resultados sugeriram que os pacientes com COVID- } 19 \text { e com DM2 têm maior } \\
\text { probabilidade de desenvolver COVID- } 19 \text { grave do que aqueles sem DM2, e que } \\
\text { a hiperglicemia está associada à linfopenia e respostas inflamatórias em pacientes } \\
\text { com COVID- } 19 \text { com DM2. }\end{array}$ \\
\hline $\begin{array}{l}\text { Dyusupov } \\
\text { a e t al., } \\
2021\end{array}$ & $\begin{array}{l}\text { Almaty, } \\
\text { Cazaquistão }\end{array}$ & Trinta e um pacientes & $\begin{array}{l}\text { Relatar as características demográficas, dados } \\
\text { clínicos e desfechos de pacientes com DM } \\
\text { COVID-19 em comparação } \\
\text { com pacientes pareados por idade e sexo sem } \\
\text { DM. }\end{array}$ & $\begin{array}{l}\text { Estudo } \\
\text { retrospectivo }\end{array}$ & $\begin{array}{l}\text { É necessário prever o monitoramento precoce do estado e cuidados de suporte } \\
\text { nesta categoria vulnerável de pacientes para permitir um melhor prognóstico. }\end{array}$ \\
\hline $\begin{array}{l}\text { Ebinger et } \\
\text { al., } 2020\end{array}$ & $\begin{array}{l}\text { Los Angeles, } \\
\text { Califórnia }\end{array}$ & $\begin{array}{l}\text { Todos os pacientes que tiveram } \\
\text { diagnóstico confirmado } \\
\text { laboratorialmente de infecçãopor } \\
\text { SARS- CoV-2 }(\mathrm{N}=442)\end{array}$ & $\begin{array}{l}\text { Determinar as características demográficas e } \\
\text { clínicas associadas ao aumento da gravidade da } \\
\text { infecção por Covid-19. }\end{array}$ & $\begin{array}{l}\text { Estudo } \\
\text { retrospectivo } \\
\text { observacional }\end{array}$ & $\begin{array}{l}\text { De todos os pacientes estudados, } 48 \% \text { necessitaram de hospitalização, } 17 \% \\
\text { necessitaram de cuidados intensivos e } 12 \% \text { necessitaram de intubação. Em } \\
\text { análises ajustadas por multivariáveis, os pacientes que requerem um nível } \\
\text { mais alto de cuidado eram mais propensos a ser mais velhos, homens, afro- } \\
\text { americanos, obeso, com diabetes mellitus e com um índice de comorbidade mais } \\
\text { alto. }\end{array}$ \\
\hline $\begin{array}{l}\text { Escobedo- } \\
\text { de la Peña } \\
\text { et al., } \\
2020\end{array}$ & México & $\begin{array}{l}\text { Todos os pacientes com teste } \\
\text { positivo para SARS- CoV-2 no } \\
\text { banco de dados institucional foram } \\
\text { incluídos para análise. }\end{array}$ & $\begin{array}{l}\text { Descrever as principais características clínicas } \\
\text { do COVID-19 na principal instituição de } \\
\text { previdência social do México, bem como a } \\
\text { contribuição das comorbidades crônicas e a } \\
\text { fração atribuível populacional a elas } \\
\text { relacionada. }\end{array}$ & $\begin{array}{l}\text { Caso controle } \\
\text { com odds ratios. }\end{array}$ & $\begin{array}{l}\text { Em } 13 \text { de novembro de } 2020,323.671 \text { indivíduos com infecção por } \\
\text { COVID- } 19 \text { foram identificados. A taxa de letalidade é maior em homens }(20,2 \%) \\
\text { do que em mulheres }(13,0 \%) \text {, e aumenta com a idade. A taxa de letalidade } \\
\text { aumentou com a presença de obesidade, hipertensão e / ou diabetes. Idade e sexo } \\
\text { foram os principais fatores de risco independentes para mortalidade, bem como } \\
\text { a presença de pneumonia, diabetes, hipertensão, obesidade, imunossupressão e } \\
\text { doença renal em estágio terminal. }\end{array}$ \\
\hline
\end{tabular}


Research, Society and Development, v. 10, n. 15, e384101522598, 2021

(CC BY 4.0) | ISSN 2525-3409 | DOI: http://dx.doi.org/10.33448/rsd-v10i15.22598

\begin{tabular}{|c|c|c|c|c|c|}
\hline $\begin{array}{l}\text { Fadini et al., } \\
\quad 2020\end{array}$ & Itália & $\begin{array}{l}\text { Dados de pacientes com COVID-19 } \\
\text { hospitalizados entre fevereiro e } \\
\text { abril de } 2020 \text { em um hospital de } \\
\text { surto. }\end{array}$ & $\begin{array}{l}\text { Investigar se o diabetes pré-existente, o } \\
\text { diabetes recém-diagnosticado e a } \\
\text { hiperglicemia na admissão estavam associadas } \\
\text { à gravidade do COVID-19, } \\
\text { independentemente de fatores de confusão. }\end{array}$ & $\begin{array}{l}\text { Estudo } \\
\text { retrospectivo }\end{array}$ & $\begin{array}{l}413 \text { indivíduos foram incluídos, } 107 \text { dos quais }(25,6 \%) \text { tinham diabetes, } \\
\text { incluindo } 21 \text { recém-diagnosticados. Pacientes com diabetes eram mais velhos e } \\
\text { tinham maior carga de comorbidades. O desfecho primário ocorreu em } 37,4 \% \\
\text { dos pacientes com diabetes em comparação com } 20,3 \% \text { naqueles sem (RR } 1,85 \text {; } \\
\text { IC 95\% 1,33-2,57; p <0,001). A associação foi mais forte para recém- } \\
\text { diagnosticados em comparação com diabetes pré-existente (RR } 3,06 \text { vs } 1,55 ; \mathrm{p}= \\
\text { 0,004). O maior nível de glicose na admissão foi associado à gravidade do } \\
\text { COVIID-19 com uma associação mais forte entre os pacientes sem diabetes em } \\
\text { comparação com aqueles com diabetes pré-existente. }\end{array}$ \\
\hline $\begin{array}{l}\text { Fox et al., } \\
2021\end{array}$ & Filadélfia & $\begin{array}{c}\text { Todos os pacientes adultos }(>18 \\
\text { anos) internados no Einstein } \\
\text { Medical Center, com diagnóstico de } \\
\text { COVID-19. }\end{array}$ & $\begin{array}{l}\text { Investigar a interação entre diabetes e COVID- } \\
19 .\end{array}$ & $\begin{array}{l}\text { Estudo } \\
\text { observacional } \\
\text { retrospectivo }\end{array}$ & $\begin{array}{l}\text { Pacientes com COVID-19 e diabetes tendem a ter doença mais grave e resultados } \\
\text { clínicos piores. Pacientes afro-americanos com diabetes não diferiram nos } \\
\text { resultados ou na gravidade da doença quando comparados aos pacientes não afro- } \\
\text { americanos. }\end{array}$ \\
\hline $\begin{array}{l}\text { Kim et al., } \\
2020\end{array}$ & $\begin{array}{c}\text { Daegu, Coréia do } \\
\text { Sul }\end{array}$ & $\begin{array}{l}\text { Pacientes adultos (idade }>18 \text { anos) } \\
\text { com COVID-19 que foi confirmado } \\
\text { por laboratório entre } 18 \text { de fevereiro } \\
\text { de } 2020 \text { e } 31 \text { de março de } 2020 .\end{array}$ & $\begin{array}{l}\text { Avaliar retrospectivamente as características } \\
\text { clínicas e os resultados de COVID- } 19 \\
\text { moderado a grave em pacientes com diabetes. }\end{array}$ & $\begin{array}{c}\text { Estudo } \\
\text { multicêntrico, } \\
\text { retrospectivo e } \\
\text { observacional }\end{array}$ & $\begin{array}{l}\text { Em comparação com o grupo sem DM }(n=847) \text {, os pacientes com DM }(n=235) \\
\text { eram mais velhos, apresentaram maior mortalidade e necessitaram de cuidados } \\
\text { mais intensivos. Mesmo após o pareamento de PS, os pacientes com DM exibiram } \\
\text { doença mais grave, e o DM permaneceu como um fator prognóstico para maior } \\
\text { mortalidade. A análise de subgrupo revelou que a presença de DM estava } \\
\text { associada a maior mortalidade, principalmente em pessoas mais velhas }(\geq 70 \\
\text { anos). }\end{array}$ \\
\hline $\begin{array}{l}\text { Lampasona } \\
\text { et al., } 2020\end{array}$ & Milão, Itália & $\begin{array}{l}\text { Pacientes adultos ( } \geq 18 \text { anos) com } \\
\text { suspeita de pneumonia por COVID- } \\
19 \text { internados entre } 25 \text { de fevereiro e } \\
19 \text { de abril de } 2020 \text {. }\end{array}$ & $\begin{array}{l}\text { Caracterizar a resposta humoral contra } \\
\text { coronavírus } 2 \text { da síndrome respiratória aguda } \\
\text { grave (SARS-CoV-2) em pacientes com } \\
\text { diabetes. }\end{array}$ & $\begin{array}{l}\text { Estudo de coorte } \\
\text { observacional }\end{array}$ & $\begin{array}{l}\text { Entre os pacientes com COVID-19 confirmado, } 139 \text { ( } 27,3 \%) \text { tinham diabetes: } 90 \\
(17,7 \%) \text { tinham diabetes diagnosticado antes da admissão hospitalar (diabetes } \\
\text { comórbido) enquanto } 49(9,6 \%) \text { tinham diabetes diagnosticado no momento da } \\
\text { admissão (diagnóstico recente). O diabetes foi associado a níveis aumentados de } \\
\text { biomarcadores inflamatórios e hipercoagulopatia, bem como leucocitose e } \\
\text { neutrofilia. O diabetes foi independentemente associado ao risco de morte, } \\
\text { mesmo após ajuste para idade, sexo e outras comorbidades relevantes. Além } \\
\text { disso, uma forte associação entreníveis mais elevados de glicose e risco de morte } \\
\text { foi documentada, independentemente do diagnóstico de diabetes. }\end{array}$ \\
\hline $\begin{array}{l}\text { Liang et al., } \\
2020\end{array}$ & China & $\begin{array}{l}\text { Pacientes adultos internados ( } 45 \\
\text { anos) do Jinling Hospital e do } \\
\text { Huoshen hill Hospital. }\end{array}$ & $\begin{array}{l}\text { Investigar as características dos achados } \\
\text { laboratoriais de pacientes com diabetes mellitus } \\
\text { tipo } 2 \text { (DM2) infectados com SARS- CoV-2. }\end{array}$ & $\begin{array}{l}\text { Estudo } \\
\text { retrospectivo }\end{array}$ & $\begin{array}{l}\text { Pacientes com DM2 infectados com SARS- CoV-2 apresentaram níveis } \\
\text { diminuídos de índice de massa corporal (IMC), linfócitos, UA e albumina e níveis } \\
\text { aumentados de PCR. A diminuição dos níveis de IMC, AU e albumina pode estar } \\
\text { associada à resposta ao estresse oxidativo e ao consumo nutricional. A diminuição } \\
\text { da contagem de linfócitos e o aumento dos níveis de PCR podem estar } \\
\text { relacionados à infecção. }\end{array}$ \\
\hline $\begin{array}{l}\text { Liu et al., } \\
2021\end{array}$ & Wuhan, China & $\begin{array}{c}\text { Todos os pacientes adultos com } \\
\text { diagnóstico confirmado de COVID- } \\
19 \text { que foram internados nas } \\
\text { enfermarias do COVID-19 de } 28 \\
\text { de janeiro de } 2020 \text { a } 4 \text { de abril de } \\
2020 . \\
\end{array}$ & $\begin{array}{l}\text { Investigar o impacto do diabetes no prognóstico } \\
\text { do COVID-19. }\end{array}$ & $\begin{array}{l}\text { Estudo } \\
\text { retrospectivo }\end{array}$ & $\begin{array}{l}\text { Diabetes, mesmo recentemente definido pelo teste de HbA1c na admissão, está } \\
\text { associado ao aumento da mortalidade em pacientes com COVID-19. A triagem } \\
\text { para diabetes não diagnosticada por medição de HbA1c deve ser considerada em } \\
\text { pacientes adultos chineses internados com COVID-19. }\end{array}$ \\
\hline $\begin{array}{l}\text { Liu et al., } \\
2020\end{array}$ & Wuhan, China & $\begin{array}{l}1.880 \text { pacientes com diagnóstico de } \\
\text { COVID-19 no Hospital } \\
\text { Leishenshan. Após excluir os casos } \\
\text { sem informação sobre a história do }\end{array}$ & $\begin{array}{l}\text { Investigar o curso clínico e os resultados de } \\
\text { pacientes com diabetes mellitus com doença } \\
\text { coronavírus } 2019 \text { (COVID-19). }\end{array}$ & $\begin{array}{l}\text { Estudo } \\
\text { observacional } \\
\text { retrospectivo }\end{array}$ & $\begin{array}{l}\text { Nossos resultados, que foram contraditórios aos de estudos anteriores com } \\
\text { grandes tamanhos de amostra, sugeriram que o diabetes não afetou } \\
\text { significativamente o prognóstico dos pacientes com COVID- 19, mas afetou } \\
\text { negativamente seu curso clínico. }\end{array}$ \\
\hline
\end{tabular}


Research, Society and Development, v. 10, n. 15, e384101522598, 2021

(CC BY 4.0) | ISSN 2525-3409 | DOI: http://dx.doi.org/10.33448/rsd-v10i15.22598

\begin{tabular}{|c|c|c|c|c|c|}
\hline & & $\begin{array}{l}\text { diabetes, a amostra final incluiu } 934 \\
\text { pacientes. }\end{array}$ & & & \\
\hline $\begin{array}{c}\text { McGurnagh } \\
\text { an et } \\
1 ., 2021^{a}\end{array}$ & Escócia & $\begin{array}{l}\text { População total da Escócia, } \\
\text { incluindo todas as pessoas com } \\
\text { diabetes que estavam vivas } 3 \\
\text { semanas antes do início da } \\
\text { pandemia na Escócia }\end{array}$ & $\begin{array}{l}\text { O objetivo do estudo foi determinar o risco } \\
\text { cumulativo de COVID-19 fatal ou tratado em } \\
\text { unidade de terapia intensiva em pessoas com } \\
\text { diabetes e compará-lo com o de pessoas sem } \\
\text { diabetes, e investigar os fatores de risco. }\end{array}$ & Estudo de coorte & $\begin{array}{l}\text { Mostramos que, entre aqueles com diabetes, o risco de doença grave varia } \\
\text { amplamente e é previsível. Essa percepção deve informar as políticas de proteção } \\
\text { e as estratégias de priorização de vacinas. }\end{array}$ \\
\hline $\begin{array}{l}\text { Mishra et } \\
\text { al., } 2020\end{array}$ & Índia & $\begin{array}{l}\text { Foram incluídos } 98 \text { pacientes } \\
\text { moderados e graves com infecção } \\
\text { por COVID- } 19 \text { em um hospital } \\
\text { COVID dedicado. O grupo de } \\
\text { estudo foi dividido em pacientes } \\
\text { com diabetes e sem diabetes. }\end{array}$ & $\begin{array}{l}\text { Estudar os níveis de dímero } \mathrm{D} \text { em pessoas com } \\
\text { diabetes em comparação com aqueles sem } \\
\text { diabetes entre pacientes com infecção por } \\
\text { COVID-19. }\end{array}$ & $\begin{array}{c}\text { Estudo } \\
\text { observacional }\end{array}$ & 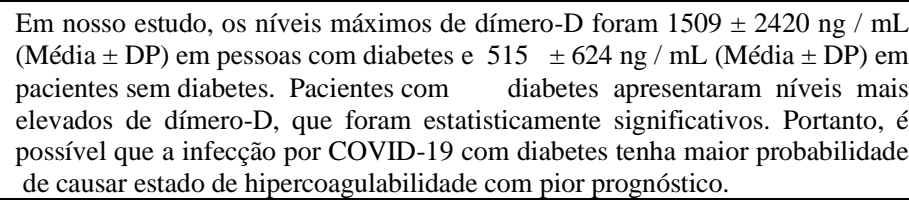 \\
\hline $\begin{array}{l}\text { Mithal et } \\
\text { al.,2020 }\end{array}$ & Índia & $\begin{array}{l}401 \text { pacientes, } 125 \text { mulheres e } 276 \\
\text { homens. }\end{array}$ & $\begin{array}{l}\text { Comparar os desfechos clínicos e inflamatórios } \\
\text { de pacientes COVID com e sem diabetes }\end{array}$ & $\begin{array}{l}\text { Estudo de caso } \\
\text { controle, } \\
\text { prospectivo, } \\
\text { observacional e } \\
\text { transversal } \\
\end{array}$ & $\begin{array}{l}\text { Foi encontrada uma alta prevalência de diabetes na população internada com } \\
\text { COVID, população essa que também apresentou desfecho mais grave e com } \\
\text { maior mortalidade. }\end{array}$ \\
\hline $\begin{array}{c}\text { Mondal et } \\
\text { al., } 2021\end{array}$ & Índia & 302 pacientes & $\begin{array}{l}\text { Estudar a incidência de cetoacidose diabética } \\
\text { em pacientes com COVID e diabéticos tipo } 2 \\
\text { recebendo glicocorticoides. }\end{array}$ & $\begin{array}{l}\text { Estudo } \\
\text { observacional, } \\
\text { prognóstico de } \\
\text { evidência }\end{array}$ & $\begin{array}{l}\text { Existe uma grande incidência de cetoacidose diabética em pacientes COVID e } \\
\text { DM2 em uso recente de glicocorticoides parenterais. }\end{array}$ \\
\hline $\begin{array}{l}\text { Odriozola } \\
\text { et al., } 2020\end{array}$ & Barcelona & $\begin{array}{l}35 \text { pacientes, } 3 \text { com DM2 e } 1 \text { com } \\
\text { DM1 }\end{array}$ & $\begin{array}{l}\text { Caracterizar a distrubuição e gravidade da } \\
\text { neuropatia sensorial em pacientes DM1 e DM2 } \\
\text { hospitalizadoscom COVID grave }\end{array}$ & Relato de caso & $\begin{array}{l}\text { Concluiu-se que a infecção grave por COVID com hipoxemia em pacientes com } \\
\text { diabetes está associada a sintomas neuropáticos e disfunção sensorial } \\
\text { generalizada. }\end{array}$ \\
\hline $\begin{array}{l}\text { O’Malley et } \\
\text { al., } 2020\end{array}$ & Estados Unidos & 113 pacientes & $\begin{array}{l}\text { Analisar características de adultos com diabetes } \\
\text { tipo } 1 \text { e sua associação com hospitalização por } \\
\text { COVID-19. }\end{array}$ & $\begin{array}{c}\text { Estudo } \\
\text { transversal } \\
\text { observacional }\end{array}$ & $\begin{array}{l}\text { Concluiu-se que o diabetes tipo } 1 \text { aumenta o risco de morbidade e mortalidade } \\
\text { por COVID, aumenta também a propensão a hospitalização. O controle glicêmico } \\
\text { e os marcadores de acesso ao tratamento são importantes medidas preventivas } \\
\text { que devem ser implantadas logo no início da infecção. }\end{array}$ \\
\hline $\begin{array}{l}\text { Satman } \\
\text { et al., } \\
2021\end{array}$ & Istambul & $\begin{array}{l}\text { Todos os pacientes adultos } \\
\text { incluindo todos os casos } \\
\text { sintomáticos de COVID-19 com ou } \\
\text { sem DM2 em Istambul }\end{array}$ & $\begin{array}{l}\text { Investigar as características clínicas e fatores de } \\
\text { risco da internação e mortalidade em pacientes } \\
\text { COVID-19 com e sem DM2 em Istambul. }\end{array}$ & $\begin{array}{l}\text { Estudo } \\
\text { retrospectivo de } \\
\text { base } \\
\text { populacional }\end{array}$ & $\begin{array}{l}\text { No grupo não-DM, enquanto o envolvimento pulmonar e a obesidade foram } \\
\text { associadas a um maior risco de hospitalização; idade, sexo masculino, linfopenia, } \\
\text { envolvimento pulmonar, insuficiência cardíaca e DRC foram associados } \\
\text { positivamente com mortalidade. No entanto, os ORs de associados comuns eram } \\
\text { menores do que o grupo DM2, e a obesidade apareceu inversamente associada à à } \\
\text { hospitalização. Além disso, pacientes com DM2 tinha doença COVID-19 clínica } \\
\text { mais grave com uma significativa maior taxa de admissão na UTI, permanência } \\
\text { na UTI por mais de } 6 \text { dias, e pneumonia. As internações hospitalares superiores } \\
\text { a } 7 \text { dias também foram mais frequentes entre os pacientes com DM2, mas a } \\
\text { diferença não atingiu significância estatística. }\end{array}$ \\
\hline $\begin{array}{l}\text { Schlöm } \\
\text { mer et } \\
\text { al., } 2020\end{array}$ & Áustria & 1 paciente, 27 anos & $\begin{array}{l}\text { Observar a progressão da infecção por COVID } \\
\text { em paciente jovem com DM1 descontrolada }\end{array}$ & Relato de caso & $\begin{array}{l}\text { Concluiu-se que, apesar de ser jovem, a paciente teve evolução grave da doença, } \\
\text { com posterior falecimento, devido a complicações por comorbidades como DM1. }\end{array}$ \\
\hline $\begin{array}{l}\text { Shang et } \\
\text { al., } 2020\end{array}$ & Wuhan & $\begin{array}{l}584 \text { pacientes, sendo } 84 \text { diabéticos e } \\
500 \text { não diabéticos }\end{array}$ & $\begin{array}{l}\text { Compreender a relação entre diabetes mellitus e } \\
\text { o prognóstico de COVID- } 19 .\end{array}$ & $\begin{array}{l}\text { Caso controle, } \\
\text { quantitativo, } \\
\text { com uso de } \\
\text { odds ratio } \\
\end{array}$ & $\begin{array}{l}\text { Concluiu-se que o diabetes é um fator de risco independente para o prognóstico } \\
\text { da COVID-19, porém atenção deve ser dada à prevenção e ao tratamento de } \\
\text { pacientes diabéticos, especialmente aqueles que necessitam de terapia com } \\
\text { insulina. }\end{array}$ \\
\hline
\end{tabular}


Research, Society and Development, v. 10, n. 15, e384101522598, 2021

(CC BY 4.0) | ISSN 2525-3409 | DOI: http://dx.doi.org/10.33448/rsd-v10i15.22598

\begin{tabular}{|c|c|c|c|c|c|}
\hline $\begin{array}{c}\text { Sun et } \\
\text { al., } 2020\end{array}$ & Estados Unidos & 3400 pacientes & $\begin{array}{l}\text { Avaliou-se o prognóstico de pacientes com } \\
\text { DM2 isoladamente ou de sua contribuição para } \\
\text { o risco de morte, síndrome do desconforto } \\
\text { respiratório agudo (SDRA), insuficiência } \\
\text { respiratória e infecção grave por COVID-19. } \\
\text { Além disso, avaliou-se os riscos associados à } \\
\text { pressão arterial elevada e glicemia de jejum. }\end{array}$ & $\begin{array}{l}\text { Estudo de caso } \\
\text { controle, } \\
\text { quantitativo } \\
\text { com uso de } \\
\text { Odds ratio }\end{array}$ & $\begin{array}{l}\text { Concluiu-se que o risco associado à hipertensão é acentuado por seu fator } \\
\text { confundido no DM2. }\end{array}$ \\
\hline $\begin{array}{l}\text { Ushigo } \\
\text { me et } \\
\text { al., } 2020\end{array}$ & Japão & 1 paciente & $\begin{array}{l}\text { Investigar a utilidade e segurança do } \\
\text { monitoramento remoto contínuo da glicose } \\
(\text { CGM) em um paciente diabético que sofre de } \\
\text { pneumonia intersticial grave causada por } \\
\text { COVID-19. }\end{array}$ & Relato de caso & $\begin{array}{l}\text { Concluiu-se que é vantajoso obter o controle da glicemia usando uma ferramenta } \\
\text { de controle remoto, prevenindo a disseminação da infecção. }\end{array}$ \\
\hline $\begin{array}{c}\text { Yan et } \\
\text { al., } 2020\end{array}$ & Wuhan & $\begin{array}{l}\text { Foram coletados dados de } 193 \\
\text { pacientes com covid-19 grave. } \\
\text { Sendo } 48 \text { pacientes com covid- } 19 \\
\text { grave tinham diabetes e } 145 \\
\text { pacientes não tinham diabetes }\end{array}$ & $\begin{array}{l}\text { Investigar as características clínicas de } \\
\text { pacientes com covid-19 grave com diabetes } \\
\text { mellitus e a associação do diabetes com o } \\
\text { desfecho em pacientes com covid- } 19 \text { grave. }\end{array}$ & $\begin{array}{l}\text { Estudo } \\
\text { unicêntrico, } \\
\text { retrospectivo e } \\
\text { observacional }\end{array}$ & $\begin{array}{l}\text { A taxa de mortalidade em pacientes com covid-19 grave com diabetes é } \\
\text { considerável. O diabetes pode aumentar o risco de morte. }\end{array}$ \\
\hline $\begin{array}{l}\text { Zhang } \\
\text { et al., } \\
2020\end{array}$ & Wuhan & 52 pacientes & $\begin{array}{l}\text { Buscar entender as características clínicas de } \\
\text { pacientes com diabetes e COVID e os fatores de } \\
\text { risco desses pacientes para desfechos ruins }\end{array}$ & $\begin{array}{l}\text { Estudo de } \\
\quad \text { coorte } \\
\text { retrospectivo de } \\
\text { centro único }\end{array}$ & $\begin{array}{l}\text { Foi descoberto que o nível elevado de cTnI era um fator de risco que prevê a } \\
\text { incidência de evolução clínica. Portanto, é recomendado que pacientes diabéticos } \\
\text { com infecção por COVID-19 tenham monitoramento vigoroso da cTnI. } \\
\text { Descobriu-se também que os } \alpha \text {-GIs podem ter um potencial efeito protetor de } \\
\text { eventos clínicos graves para esses pacientes. }\end{array}$ \\
\hline $\begin{array}{l}\text { Zhang } \\
\text { et al., } \\
2020\end{array}$ & Wuhan & 27 pacientes & $\begin{array}{l}\text { Descrever as características dos pacientes com } \\
\text { COVID-19 com diabetes tipo } 2 \text { e analisar os } \\
\text { fatores de risco para a gravidade. }\end{array}$ & $\begin{array}{l}\text { Caso controle } \\
\text { com uso de } \\
\text { odds ratio }\end{array}$ & $\begin{array}{l}\text { Concluiu-se que pacientes diabéticos tipo } 2 \text { eram mais suscetíveis a COVID- } 19 \\
\text { do que a população geral, o que pode estar associado a hiperglicemia e } \\
\text { dislipidemia }\end{array}$ \\
\hline
\end{tabular}

Fonte: Autores. 
Durante o estudo foi possível observar a forte correlação entre o mal prognóstico de COVID-19 e Diabetes tipo 1 ou 2 (Zhang et al., 2020) (Schlömmer et al., 2020). Visto que o diabetes imunocompromete o corpo humano, entende-se que uma infecção secundária a COVID - 19 seja mais persistente e letal (Akter et al, 2020).

Com isso, este imunocomprometimento em pacientes diabéticos resulta na exacerbação da resposta inflamatória, com o aumento de níveis de leucócitos, contagem de neutrófilos, proteína de reação $\mathrm{C}$ de alta sensibilidade, procalcitonina, ferritina, receptor de interleucina (IL) 2, IL-6, IL-8, fator de necrose tumoral $\alpha$, D-dímero, fibrinogênio, desidrogenase láctica e peptídeo natriurético N-terminal pró-cérebro e baixa resposta de linfócitos. Desse modo, a infecção em pacientes DM pode levar sintomas exacerbados, como febre persistente e alta, dificuldade respiratória significativamente aumentada, com grandes chances de progressão agudizada, podendo resultar em pneumonia grave e síndrome da aflição respiratória, cursando com necessidade de cuidados intensivos. Além disso, pacientes internados por Covid e portadores de diabetes, tem altas taxas de cetoacidose diabética e hiperglicemia, o que agrava consideravelmente a condição do paciente (O’Malley et al, 2020)

No que concerne a comorbidades associadas ao diabetes em pacientes COVID-19, tem-se um maior índice de doenças cardiovasculares, câncer, doenças respiratórias e doença renal crônica. (Akter et al., 2020) (Yan et al., 2020) Desse modo, de acordo com estudos anteriores e presente investigação, foi possível inferir que o impacto depletor de COVID-19 é maior em pacientes comórbidos do que aqueles que não têm comorbidades (O’Malley et al., 2020); (Satman et al., 2021); (Schlömmer et al., 2020).

Foi identificado estatisticamente que a maior parte dos pacientes com alto risco de morte são idosos e homens, os quais apresentam pior resposta inflamatória grave, disfunção pulmonar com a redução do volume expiratório forçado, capacidade vital forçada, associado a cardiopatia, doença microvascular diabética (com função renal mais comprometida e retinopatia) doença hepática, renal e de coagulação. Estes pacientes também apresentavam pior controle glicêmico e eram mais propensos a ter cetoacidose diabética ou hospitalização por hipoglicemia anterior (Yan et al., 2020) (McGurnaghan et al., 2021)

Considerando o que foi dito, pacientes DM e COVID tem maiores chances de contrair doenças infecciosas, como pneumonia, síndrome respiratória aguda grave (SARS) e Síndrome Respiratória do Oriente Médio (MERS). Visto isso, estudos enfocaram em como melhorar o prognóstico do paciente nessa situação, e foi encontrado que o indivíduo com a glicose controlada tem maior taxa de sobrevida.

Encontrou-se no caso específico da Diabetes tipo 1, os dados de forma limitada. Um estudo feito comparando pacientes com Diabetes tipo 1 e pacientes sem diabetes conhecida, revelou que aqueles com DM1 associada a doenças cardiovasculares, sexo, idade, etnia, índice de massa corporal, localização geográficas tiveram considerável aumento na morbimortalidade. (O’Malley, et al, 2020)

Os fatores de risco mais comuns para estes pacientes giram em torno de idade avançada, etnia negra, uso de seguro de saúde, HbA1c elevada e hipertensão. Nesse recorte, gênero não foi considerado um fator de rico. Nesse mesmo recorte, foi claro o desfecho de aumento de morbimortalidade em pacientes DM1 associada a Sars-Cov-2, além de, quanto mais comórbido o paciente, maior a chance de morte. Entendeu-se também que ferramentas importantíssimas para um melhor desfecho são o controle glicêmico inicial e constante, e favoráveis condições hospitalares. (Schlömmer et al., 2020) (O’Malley et al, 2020)

\section{Conclusão}

Conclui-se, em virtude dos estudos abordados, que existe uma maior necessidade de respostas inflamatórias por consequência da agressão virulenta em pacientes DM, já que os mesmos possuem menor ação linfocitária, diminuição da função hepática e considerável hiperglicemia quando comparados a pacientes não diabéticos, levando ao agravamento do quadro e aumento da morbimortalidade dos pacientes. Ademais, evidenciou-se que quanto mais comorbidades, além da DM, o paciente 
apresenta, pior é o prognóstico, especialmente se essas comorbidades são doenças crônicas, como condições cardiovasculares, a exemplo da hipertensão, e doença renal crônica.

Entende-se que, com o avanço da pandemia, é necessário o cuidado mais acurado com o paciente, em virtude das variantes de risco para COVID-19, e esse cuidado deve ser intensificado principalmente em diabéticos, adaptando as medidas de prevenção necessárias, como controle glicêmico rigoroso e maior atenção a evolução do quadro. Ademais, urge que as campanhas de vacinação sigam em progressão, e que a população diabética entenda o risco que corre e possa se conscientizar da necessidade de imunização.

Essa revisão pode abrir portas para novas discussões a respeito dessa temática. Estudos clínicos envolvendo análises volumes e capacidades pulmonares, e quantificação de parâmetros laboratoriais de DM, ambos associados ao COVID - 19 pode ser uma vertente para estudos posteriores que poderão trazer esclarecimentos mais concretos nesse cenário.

\section{Referências}

Abbas, H. M., Nassir, K. F., Al Khames Aga, Q. A., Al-Gharawi, A. A., Rasheed, J. I., AL-Obaidy, M. W., \& Al Khames Aga, L. A. (2021). Presenting the characteristics, smoking versus diabetes, and outcome among patients hospitalized with COVID-19. Journal of medical virology, 93(3), $1556-1567$.

Akter, F., Mannan, A., Mehedi, H. H., Rob, M. A., Ahmed, S., Salauddin, A., \& Hasan, M. M. (2020). Clinical characteristics and short term outcomes after recovery from COVID-19 in patients with and without diabetes in Bangladesh. Diabetes \& Metabolic Syndrome: Clinical Research \& Reviews, 14(6), 20312038 .

Akbariqomi, M., Hosseini, M. S., Rashidiani, J., Sedighian, H., Biganeh, H., Heidari, R., \& Kooshki, H. (2020). Clinical characteristics and outcome of hospitalized COVID-19 patients with diabetes: A single-center, retrospective study in Iran. diabetes research and clinical practice, $169,108467$.

Alam, M. R., Kabir, M. R., \& Reza, S. (2021). Comorbidities might be a risk factor for the incidence of COVID-19: Evidence from a web- based survey. Preventive Medicine Reports, 21, 101319.

Al-Salameh, A., Lanoix, J. P., Bennis, Y., Andrejak, C., Brochot, E., Deschasse, G., \& Lalau, J. D. (2021). Characteristics and outcomes of COVID-19 in hospitalized patients with and without diabetes. Diabetes/metabolism research and reviews, 37(3), e3388.

Alzaid, F., Julla, J. B., Diedisheim, M., Potier, C., Potier, L., Velho, G., \& Gautier, J. F. (2020). Monocytopenia, monocyte morphological anomalies and hyperinflammation characterise severe COVID-19 in type 2 diabetes. EMBO molecular medicine, 12(10), e13038.

Arango-Guerra, P., Moncayo-Viveros, J. G., Correa-Londoño, N., García-Roncallo, A., Ramírez-Escobar, L., Sánchez-Moreno, A. M., \& Moreno-Bedoya, S. (2021). Crisis hiperglucémica concomitante con infección por SARS-CoV-2: una serie de casos. Acta Colombiana de Cuidado Intensivo.

Barron, E., Bakhai, C., Kar, P., Weaver, A., Bradley, D., Ismail, H., \& Valabhji, J. (2020). Associations of type 1 and type 2 diabetes with COVID-19- related mortality in England: a whole-population study. The lancet Diabetes \& endocrinology, 8(10), 813-822.

Bhatti, R., Khamis, A. H., Khatib, S., Shiraz, S., \& Matfin, G. (2020). Clinical Characteristics and Outcomes of Patients With Diabetes Admitted for COVID19 Treatment in Dubai: Single-Centre Cross-Sectional Study. JMIR public health and surveillance, 6(4), e22471.

Chan, K. H., Thimmareddygari, D., Ramahi, A., Atallah, L., Baranetsky, N. G., \& Slim, J. (2020). Clinical characteristics and outcome in patients with combined diabetic ketoacidosis and hyperosmolar hyperglycemic state associated with COVID- 19: a retrospective, hospital-based observational case series. Diabetes research and clinical practice, 166, 108279.

Chen, Q., Zheng, Z., Zhang, C., Zhang, X., Wu, H., Wang, J., \& Zheng, C. (2020). Clinical characteristics of 145 patients with corona virus disease 2019 (COVID-19) in Taizhou, Zhejiang, China. Infection, 48(4), 543-551.

Cheng, Y., Yue, L., Wang, Z., Zhang, J., \& Xiang, G. (2021). Hyperglycemia associated with lymphopenia and disease severity of COVID-19 in type 2 diabetes mellitus. Journal of Diabetes and its Complications, 35(2), 107809.

Dyusupova, A., Faizova, R., Yurkovskaya, O., Belyaeva, T., Terekhova, T., Khismetova, A., \& Glushkova, N. (2021). Clinical characteristics and risk factors for disease severity and mortality of COVID-19 patients with diabetes mellitus in Kazakhstan: A nationwide study. Heliyon, 7(3), e06561.

Ebinger, J. E., Achamallah, N., Ji, H., Claggett, B. L., Sun, N., Botting, P., \& Cheng, S. (2020). Pre-existing traits associated with Covid- 19 illness severity. PloS one, 15(7), e0236240.

Escobedo-de la Peña, J., Pacheco, R. A. R., de Jesús Ascencio-Montiel, I., González-Figueroa, E., Fernández-Gárate, J. E., Medina-Gómez, O. S., \& BorjaAburto, V. H. (2020). Hypertension, diabetes and obesity, major risk factors for death in patients with COVID-19 in Mexico. Archives of Medical Research.

Fadini, G. P., Morieri, M. L., Boscari, F., Fioretto, P., Maran, A., Busetto, L., \& Vettor, R. (2020). Newly- diagnosed diabetes and admission hyperglycemia predict COVID-19 severity by aggravating respiratory deterioration. Diabetes research and clinical practice, $168,108374$.

Fox, T., Ruddiman, K., Lo, K. B., Peterson, E., DeJoy, R., Salacup, G., \& Patarroyo-Aponte, G. (2021). The relationship between diabetes and clinical outcomes in COVID-19: a single-center retrospective analysis. Acta Diabetologica, 58(1), 33-38. 
Kim, M. K., Jeon, J. H., Kim, S. W., Moon, J. S., Cho, N. H., Han, E., \& Lee, J. H. (2020). The clinical characteristics and outcomes of patients with moderateto-severe coronavirus disease 2019 infection and diabetes in Daegu, South Korea. Diabetes \& metabolism journal, 44(4), 602.

Lampasona, V., Secchi, M., Scavini, M., Bazzigaluppi, E., Brigatti, C., Marzinotto, I., \& Piemonti, L. (2020). Antibody response to multiple antigens of SARSCoV-2 in patients with diabetes: an observational cohort study. Diabetologia, 63(12), 2548-2558.

Liang, J. J., Liu, J., Chen, Y., Ye, B., Li, N., Wang, X., \& Shao, J. (2020). Characteristics of laboratory findings of COVID-19 patients with comorbid diabetes mellitus. Diabetes research and clinical practice, $167,108351$.

Liu, Y., Lu, R., Wang, J., Cheng, Q., Zhang, R., Zhang, S., \& Hong, T. (2021). Diabetes, even newly defined by HbA1c testing, is associated with an increased risk of in-hospital death in adults with COVID- 19. BMC Endocrine Disorders, 21(1), 1-10.

Liu, Z., Li, J., Huang, J., Guo, L., Gao, R., Luo, K., \& Wu, X. (2020). Association Between Diabetes and COVID-19: A Retrospective Observational Study With a Large Sample of 1,880 Cases in Leishenshan Hospital, Wuhan. Frontiers in endocrinology, 11, 478.

McGurnaghan, S. J., Weir, A., Bishop, J., Kennedy, S., Blackbourn, L. A., McAllister, D. A., \& Health Protection Study Group. (2021). Risks of and risk factors for COVID-19 disease in people with diabetes: a cohort study of the total population of Scotland. The Lancet Diabetes \& Endocrinology, 9(2), 82-93.

Mishra, Y., Pathak, B. K., Mohakuda, S. S., Tilak, T. V. S. V. G. K., Sen, S., Harikrishnan, P., \& Singh, A. R. (2020). Relation of D-dimer levels of COVID19 patients with diabetes mellitus. Diabetes \& Metabolic Syndrome: Clinical Research \& Reviews, 14(6), 1927-1930.

Mithal, A., Jevalikar, G., Sharma, R., Singh, A., Farooqui, K. J., Mahendru, S., \& Budhiraja, S. (2021). High prevalence of diabetes and other comorbidities in hospitalized patients with COVID-19 in Delhi, India, and their association with outcomes. Diabetes \& Metabolic Syndrome: Clinical Research \& Reviews, $15(1), 169-175$

Mondal, S., DasGupta, R., Lodh, M., Gorai, R., Choudhury, B., Hazra, A. K., \& Ganguly, A. (2021). Predictors of new- onset diabetic ketoacidosis in patients with moderate to severe COVID-19 receiving parenteral glucocorticoids: A prospective single- centre study among Indian type 2 diabetes patients. Diabetes \& Metabolic Syndrome: Clinical Research \& Reviews, 15(3), 795-801.

Odriozola, A., Ortega, L., Martinez, L., Odriozola, S., Torrens, A., Corroleu, D., \& Malik, R. A. (2021). Widespread sensory neuropathy in diabetic patients hospitalized with severe COVID-19 infection. Diabetes research and clinical practice, 172, 108631.

O’Malley, G., Ebekozien, O., Desimone, M., Pinnaro, C. T., Roberts, A., Polsky, S., \& Levy, C. J. (2021). COVID-19 Hospitalization in Adults with Type 1 Diabetes: Results from the T1D Exchange Multicenter Surveillance Study. The Journal of Clinical Endocrinology \& Metabolism, 106(2), e936-e942.

Satman, I., Demirci, I., Haymana, C., Tasci, I., Salman, S., Ata, N., \& Sonmez, A. (2021). Unexpectedly lower mortality rates in COVID- 19 patients with and without type 2 diabetes in Istanbul. Diabetes Research and Clinical Practice, 174, 108753.

Schlömmer, C., Dünser, M. W., Antlanger, M., Paar, C., Winkler, M., Meier, J., \& Salzer, H. J. (2020). Fatal case of COVID-19 in a 27-year- old woman with diabetes mellitus. Wiener klinische Wochenschrift, 132(21), 695-696.

Shang, J., Wang, Q., Zhang, H., Wang, X., Wan, J., Yan, Y., \& Lin, J. (2021). The relationship between diabetes mellitus and COVID-19 prognosis: a retrospective cohort study in Wuhan, China. The American journal of medicine, 134(1), e6-e14.

Sun, Y., Guan, X., Jia, L., Xing, N., Cheng, L., Liu, B., \& He, K. (2021). Independent and combined effects of hypertension and diabetes on clinical outcomes in patients with COVID-19: A retrospective cohort study of Huoshen Mountain Hospital and Guanggu Fangcang Shelter Hospital. The Journal of Clinical Hypertension, 23(2), 218-231

Ushigome, E., Yamazaki, M., Hamaguchi, M., Ito, T., Matsubara, S., Tsuchido, Y., \& Fukui, M. (2021). Usefulness and safety of remote continuous glucose monitoring for a severe COVID-19 patient with diabetes. DiabetesTechnology \& Therapeutics, 23(1), 78-80.

Yan, Y., Yang, Y., Wang, F., Ren, H., Zhang, S., Shi, X., \& Dong, K. (2020). Clinical characteristics and outcomes of patients with severe covid- 19 with diabetes. BMJ open diabetes research and care, $8(1)$, e001343.

Zhang, N., Wang, C., Zhu, F., Mao, H., Bai, P., Chen, L. L., \& Zhou, M. (2020). Risk factors for poor outcomes of diabetes patients with COVID-19: a singlecenter, retrospective study in early outbreak in China. Frontiers in endocrinology, 11 .

Zhang, Q., Wei, Y., Chen, M., Wan, Q., \& Chen, X. (2020). Clinical analysis of risk factors for severe COVID-19 patients with type 2 diabetes. Journal of Diabetes and its Complications, 34(10) 